\title{
EFEITOS ADVERSOS À POLIQUIMIOTERAPIA EM PACIENTES COM HANSENÍASE ATENDIDOS NAS UNIDADES BÁSICAS DE SAÚDE
}

Tadeu Augusto Filho ${ }^{1}$, Marilena Maria de Souza², Vagner Alexandre de Sousa ${ }^{3}$, Ana Emília Formiga Marques ${ }^{4}$, Francisca Dara Augusto de Sousa ${ }^{5}$,

${ }^{1}$ Curso de Bacharelado em Enfermagem, Unidade Acadêmica de Saúde, Universidade Federal de Campina Grande, Cajazeiras-PB, Brasil.

${ }^{2}$ Prof ${ }^{a}$ Unidade Acadêmica de Saúde, Universidade Federal de Campina Grande, CajazeirasPB, Brasil.

${ }^{3}$ Curso de Bacharelado em Farmácia, Unidade Acadêmica de Saúde, Faculdade São Francisco da Paraíba, Cajazeiras-PB, Brasil.

${ }^{4}$ Profa Unidade Acadêmica de Saúde, Faculdade São Francisco da Paraíba, Cajazeiras-PB, Brasil.

${ }^{5}$ Curso de Pós-graduação em Farmácia Hospitalar, Unidade Acadêmica de Saúde, Faculdade Santa Maria, Cajazeiras-PB, Brasil.

Email para correspondência: anaeformiga@gmail.com

\section{Resumo}

\begin{abstract}
A hanseníase é uma doença crônica granulomatosa, proveniente de infecção causada pelo bacilo Mycobacterium leprae. Desde a década de 1980, foram preconizados pela Organização Mundial de Saúde a Poliquimioterapia para tratamento da doença, sendo composta pela combinação das drogas dapsona, clofazimina e rifampicina. Diante disso, objetivou-se verificar os efeitos adversos às drogas usadas e irregularidades no tratamento da hanseníase e a ocorrência na mudança de esquema terapêutico, a partir da revisão dos prontuários de pacientes tratados nas Unidades Básicas de Saúde do município de CajazeirasPB. Estudo de caráter retrospectivo e documental, com abordagem quantitativa. Foram coletados dados dos prontuários de 48 pacientes que concluíram o tratamento para hanseníase entre janeiro de 2013 a maio de 2016. Os efeitos cutâneos relacionados à clofazimina foram os mais frequentes dentre os efeitos adversos, tais como a hiperpigmentação da pele $(45,8 \%)$ e ictiose/xerose $(37,5 \%)$. Dentre as reações adversas encontradas em exames laboratoriais, se destaca a anemia (25\%), associada ao uso da dapsona. O surgimento de reações adversas medicamentosas interferiu na adesão ao tratamento, no qual foi confirmado o abandono de dois pacientes. Sendo assim, faz-se necessário que haja uma busca ativa eficaz dos pacientes, para prevenir casos de abandono.
\end{abstract}

Palavras-chave: Hanseníase. Efeitos Adversos. Poliquimioterapia. Abandono. 


\begin{abstract}
Leprosy is a chronic granulomatous disease, caused by infection caused by the bacillus Mycobacterium leprae. Since the 1980s, polychemotherapy for the treatment of the disease has been recommended by the World Health Organization, consisting of the combination of dapsone, clofazimine and rifampicin. Therefore, the objective was to verify the adverse effects of the drugs used and irregularities in the treatment of leprosy and the occurrence of changes in the therapeutic scheme, based on the review of the medical records of patients treated in the Basic Health Units of the municipality of Cajazeiras-PB. Retrospective and documentary study, with a quantitative approach. Data were collected from the medical records of 48 patients who completed treatment for leprosy between January 2013 and May 2016. Cutaneous effects related to clofazimine were the most frequent of the adverse effects, such as skin hyperpigmentation $(45.8 \%)$ and ichthyosis / xerosis (37.5\%). Among the adverse reactions found in laboratory tests, anemia (25\%), associated with the use of dapsone, stands out. The emergence of adverse drug reactions interfered with treatment adherence, in which the abandonment of two patients was confirmed. Therefore, it is necessary to have an effective active search for patients, to prevent cases of abandonment.
\end{abstract}

Keywords: Leprosy. Adverse Effects. Polychemotherapy. Abandonment.

\title{
1 Introdução
}

A hanseníase é uma doença crônica granulomatosa, com alta infectividade e baixa patogenicidade, causada pelo Mycobacterium leprae, que acomete pele e nervos periféricos, possuindo alto potencial incapacitante, que está diretamente relacionado à imunogenicidade do bacilo (BRASIL, 2019). Esta doença representa um importante problema de saúde pública em vários países no mundo (VIRMOND, 2013).

Para a Organização Mundial de Saúde (OMS) foram reportados casos de hanseníase em 105 países e territórios, durante o primeiro quadrimestre de 2012, com uma prevalência mundial de 181.941 casos em tratamento (BRASIL, 2014). O Brasil ocupa a segunda posição em número de casos novos de hanseníase, no ano de 2014, com 31.064 casos (15,46\%) (WHO, 2015). A região Nordeste apresentou um padrão de alta endemicidade, com média de 23,79/100.000 habitantes (BRASIL, 2014).

O estado da Paraíba apresentou uma redução de prevalência em 2010 com 1,5 casos/10.000 habitantes, considerou-se um estado com padrão de média endemicidade. De um total de 223 municípios, 129 não notificaram casos de hanseníase em 2010, 14 municípios hiperendêmicos, dos quais, apenas 3 tiveram mais de 10 casos diagnosticados. O município de Cajazeiras 
apresentou neste mesmo ano o maior coeficiente de detecção, com 107,8 casos/100.000 habitantes (BRASIL, 2011).

A atual Estratégia Global para Hanseníase 2016-2020 tem como finalidade a detecção precoce da doença, tratamento imediato visando à prevenção de incapacidades e a redução da transmissão da infecção na comunidade, tendo como inovação, o foco em aspectos humanos e sociais que afetem o controle da hanseníase (WHO, 2016).

O tratamento da hanseníase é feito a partir da poliquimioterapia (PQT/OMS), constituída pela associação dos medicamentos rifampicina, dapsona e clofazimina, sendo administrada a partir de esquema padrão, de acordo com a classificação operacional do paciente. Os medicamentos utilizados na poliquimioterapia podem causar vários efeitos adversos (BRASIL, 2017). O tratamento longo, os efeitos adversos e episódios reacionais da hanseníase podem levar o paciente à interrupção ou abandono do tratamento, constituindo-se em um grande desafio para os profissionais de saúde (SOUZA et al., 2009).

Estudo realizado por Kar et al. (2010), no distrito de Kamrup, localizado em Assam (Índia), sobre adesão ao tratamento de hanseníase, foi composto pela amostra de 1.020 pacientes, no qual as reações adversas aos medicamentos das drogas PQT foram a segunda maior causa $(25,98 \%)$ de abandono ao tratamento, superado apenas para motivos ocupacionais (33,07\%), quando os pacientes não compareciam ao serviço de saúde para não perderem as horas de trabalho.

Diante do exposto, esse estudo se justifica, tendo em vista que o tratamento da doença é longo, com múltiplas drogas, elevando o risco de reações adversas, algumas delas, potencialmente graves, o que implica na necessidade de conhecer as reações adversas, que implica no diagnóstico e manejo de tais efeitos adversos, evitando suspensão inadequada dessas drogas, o que poderia levar à resistência medicamentosa e ao fracasso terapêutico.

Assim, objetivou-se verificar os efeitos adversos às drogas usadas e irregularidades no tratamento da hanseníase e a ocorrência na mudança de 
esquema terapêutico, a partir da revisão dos prontuários de pacientes tratados nas Unidades Básicas de Saúde do município de Cajazeiras-PB.

\section{Metodologia}

\subsection{Tipo de pesquisa}

Trata-se de um estudo retrospectivo e documental, com abordagem quantitativa.

\subsection{Local da pesquisa}

A pesquisa foi realizada nas Unidades Básicas de Saúde (UBS) pertencentes ao município de Cajazeiras-PB: UBS Posto de Assistência Primária a Saúde (PAPS) situada na Rua Tenente Aquino Albuquerque, UBS Amélio Estrela situada na Rua Vitória Bezerra, UBS Sol Nascente situada na Rua Raimundo Moesia Rolim, UBS Simão de Oliveira situada na Rua Coronel Juvêncio Carneiro e UBS João Bosco Bragado primeiro para Barreto situada na Rua Luiz Paulo e Silva.

O município de Cajazeiras-PB está situado na região oeste do estado da Paraíba, localizado à aproximadamente 468 quilômetros da capital estadual, João Pessoa. Possui atualmente 24 UBS credenciadas pelo Ministério da Saúde (MS), com uma cobertura populacional de cerca de 60 mil habitantes. A escolha das UBS para realização desta pesquisa se deve ao fato de ser porta de entrada principal das redes de atenção à saúde, e por estarem localizadas em um município hiperendêmico para hanseníase.

\subsection{População e amostra}

A população deste estudo foi composta por prontuários de pacientes que concluíram o tratamento para hanseníase pelo esquema padrão PQT/OMS nas unidades básicas de saúde PAPS, Amélio Estrela, Sol Nascente, Simão de Oliveira e Dom Bosco, do município de Cajazeiras-PB.

Já a amostra foi composta por 48 prontuários de pacientes que concluíram o tratamento para hanseníase nas UBS e que atenderam aos critérios de inclusão e exclusão. 


\subsection{Critérios}

Os critérios de inclusão foram: ter concluído o tratamento com PQT para hanseníase pelo esquema padrão, preconizado pelo MS, referente ao período de janeiro de 2013 a maio de 2016. E excluídos os prontuários sem informações necessárias e que não se encontravam nos arquivos das referidas UBS.

\subsection{Coleta de dados}

A coleta de dados foi feita entre agosto e setembro de 2016, por estudantes do curso de enfermagem a partir de informações obtidas pela revisão de prontuários de pacientes tratados com Poliquimioterapia Paucibacilar PQT/PB e Poliquimioterapia Multibacilar PQT/MB para hanseníase nas UBS préestabelecidas.

Também foi elaborado um formulário de investigação de eventos adversos para organizar os dados obtidos nos prontuários, composto pelas variáveis: características demográficas (sexo e idade), reações adversas (clínicas e laboratoriais), correlação entre as reações adversas e o tratamento (PQT e mudança de esquema).

Após a coleta, os dados obtidos foram processados quantitativamente e, posteriormente, apresentados em tabelas e gráficos, para correlacionar as variáveis pesquisadas. Todos os dados obtidos foram analisados de forma descritiva, no qual recorreu-se à literatura pertinente.

\subsection{Considerações éticas}

Este projeto foi aprovado pelo Comitê de Ética da Universidade Federal de Campina Grande, campus Cajazeiras, parecer no 1.703.202, conforme estabelecem as Diretrizes e Normas Regulamentadoras de Pesquisas Envolvendo Seres Humanos, adequado a Resolução 466/2012 em seus aspectos éticos (BRASIL, 2012).

\section{Resultados e discussão}

Foram coletados dados de 48 prontuários de pacientes com hanseníase de UBS, no município de Cajazeiras-Paraíba. Para tanto, foi realizada análise quantitativa, com caracterização demográfica, epidemiológica e clínica dos 
pacientes que concluíram o tratamento; análise de prontuários de tratamento para quantificar e tipificar efeitos adversos, associando-os a mudança de esquema terapêutico e adesão ao tratamento.

Tabela 1: Caracterizações demográfica, epidemiológica e clínicas dos indivíduos estudados.

\begin{tabular}{lcr}
\hline Categorias & & $\mathbf{n}(\%)$ \\
\hline Idade (anos) & $0-14$ & $1(2,1)$ \\
& $15-40$ & $21(43,8)$ \\
& $41-65$ & $16(33,3)$ \\
& $>65$ & $10(20,8)$ \\
\hline Sexo & Masculino & $30(62,5)$ \\
\hline Formas Clínicas & Feminino & $18(37,5)$ \\
& Hanseníase Indeterminada & $13(27,1)$ \\
& Hanseníase Tuberculóide & $13(27,1)$ \\
& Hanseníase Dimorfa & $16(33,3)$ \\
\hline Classificação & Hanseníase Virchowiana & $6(12,5)$ \\
Operacional & Paucibacilares & $26(54,2)$ \\
\hline Baciloscopia & Multibacilares & $22(45,8)$ \\
Admissional & Positiva & $11(22,9)$ \\
& Negativa & $35(72,9)$ \\
& Não realizada & $2(4,2)$ \\
\hline
\end{tabular}

Dos 48 pacientes estudados, a faixa etária predominante foi entre 15 e 40 anos, com $21(43,8 \%)$ indivíduos. O sexo masculino predominou entre os gêneros, com um total de 30 (62,5\%). A forma clínica mais frequente foi a HD, com $16(33,3 \%)$ casos. Quanto à classificação operacional, 26 (54,2\%) pacientes foram classificados como Paucibacilar, representando a maioria. $\mathrm{Na}$ realização da baciloscopia admissional, 35 (72,9\%) tiveram resultado negativo. Em 2 (4,2\%) casos não foi realizado o exame baciloscópico (Tabela 1).

Estudo realizado por Miranzi et al. (2010), sobre o perfil epidemiológico de hanseníase em município brasileiro, com 455 casos apontou que 252 (55,4\%) dos pacientes eram homens. A forma clínica prevalente foi adimorfa $(69,1 \%)$, 
apesar de que na classificação operacional, a forma multibacilar predominou com $396(87,1 \%)$ casos, divergindo deste estudo apenas na classificação operacional.

A alta prevalência de casos diagnosticados tardiamente, como por exemplo, a forma clínica dimorfa, constatada neste estudo, é um dado preocupante, visto que o paciente tem maior tendência a desenvolver incapacidades físicas, dentre outras complicações. Em estudo realizado por Mendes et al. (2014), na macrorregião de Barbacena-MG, sobre caracterização clinico-epidemiológica da hanseníase,com 114 pacientes, avaliou-se a relação entre classificação operacional (PB e MB) e grau de incapacidade física. No grau 0 houve predominância dos pacientes Paucibacilares com 56,4\% dos casos. Nos graus I e II, a maioria eram Multibacilares com frequência de 51,1\% e $24,4 \%$, respectivamente. Muito embora neste estudo tenha havido predominância de pacientes Paucibacilares o número de pacientes com a forma clínica dimorfa, ou seja, MBS, não deixa de ser relevante.

A alta taxa de resultados negativos na baciloscopia admissional, que neste estudo esteve presente em 35 (72,9\%) pacientes, é justificada pela prevalência de Paucibacilares (resultado negativo), além da contribuição, em parte, da forma clínica dimorfa (resultado negativo ou positivo) (BRASIL, 2014).

Este estudo apresentou uma frequência relativamente baixa de efeitos adversos em relação a outros estudos devido às limitações impostas pelo desenho retrospectivo pesquisado. A utilização de prontuários de tratamento, que não são estruturados para fornecer dados específicos quanto aos efeitos adversos, corroborou para este resultado, sugerindo que possa haver vários eventos adversos não constados por falta de registro efetivo.

As reações adversas foram organizadas e quantificadas em eventos clínicos e laboratoriais de acordo com a amostra. Os efeitos adversos clínicos foram categorizados segundo alterações específicas, como: alterações cutâneas, neurossensoriais, gastrointestinais, e outros, para melhor esclarecimento, demonstrada na Tabela 2. 
Tabela 2: Frequência dos efeitos adversos clínicos dos participantes.

\begin{tabular}{|c|c|c|}
\hline \multicolumn{3}{|c|}{ Reações Adversas Clínicas } \\
\hline Categorias & Descrição & $n(\%)$ \\
\hline \multirow[t]{5}{*}{ Alterações cutâneas } & Hiperpigmentação da pele & $22(45,8)$ \\
\hline & Ictiose/Xerose & $18(37,5)$ \\
\hline & Metaemoglobinemia & $1(2,1)$ \\
\hline & Quelite angular & $1(2,1)$ \\
\hline & Erupção acneiforme & $1(2,1)$ \\
\hline Alterações & Náuseas & $11(22,9)$ \\
\hline \multirow[t]{5}{*}{ gastrointestinais } & Dor epigástrica & $5(10,4)$ \\
\hline & Dor abdominal & $3(6,2)$ \\
\hline & Hiporexia & $3(6,2)$ \\
\hline & Diarreia & $2(4,2)$ \\
\hline & Vômitos & $2(4,2)$ \\
\hline Alterações & Vertigem & $7(14,6)$ \\
\hline \multirow[t]{4}{*}{ neurossensoriais } & Insônia & $4(8,3)$ \\
\hline & Cefaléia & $2(4,2)$ \\
\hline & Alterações do humor & $3(6,2)$ \\
\hline & Tremores & $1(2,1)$ \\
\hline \multirow[t]{3}{*}{ Outros } & Câimbras & $3(6,2)$ \\
\hline & Fadiga & $2(4,2)$ \\
\hline & Perda de peso & $2(4,2)$ \\
\hline
\end{tabular}

$\mathrm{Na}$ tabela 2, as alterações cutâneas relacionadas à clofazimina, como a hiperpigmentação de pele e a ictiose/xerose se sobressaíram dentre as demais, com frequência de $45,8 \%$ e $37,5 \%$, respectivamente. Pele seca e ictiósica ocorrem com bastante frequência no tratamento com clofazimina, variando, conforme o tipo de pele e a dose a ser administrada (FRANCO, 2014).

No estudo realizado por Maia et al.(2013), na cidade de Manaus, norte brasileiro sobre efeitos adversos das drogas utilizadas na quimioterapia 
alternativa, foi observado hiperpigmentação cutânea relacionada à clofazimina em $100 \%$ dos pacientes.

As alterações gastrointestinais tiveram uma frequência relevante, como as náuseas $(22,9 \%)$ e a dor epigástrica (10,4\%). As alterações neurossensoriais com maior destaque foram a vertigem $(14,6 \%)$ e a insônia (8,3\%).

Como evento adverso relacionado ao uso da rifampicina, que é feito mensalmente, pode ser destacado algumas das alterações gastrintestinais, como a hiporexia $(6,2 \%)$ e diarreia $(4,2 \%)$, confirmando a baixa frequência desses efeitos nesse estudo. Este achado está de acordo com dois estudos realizados a cerca dessa temática (ROQUE e MELO, 2010).

Gráfico 1: Frequência dos efeitos adversos laboratoriais dos pacientes.

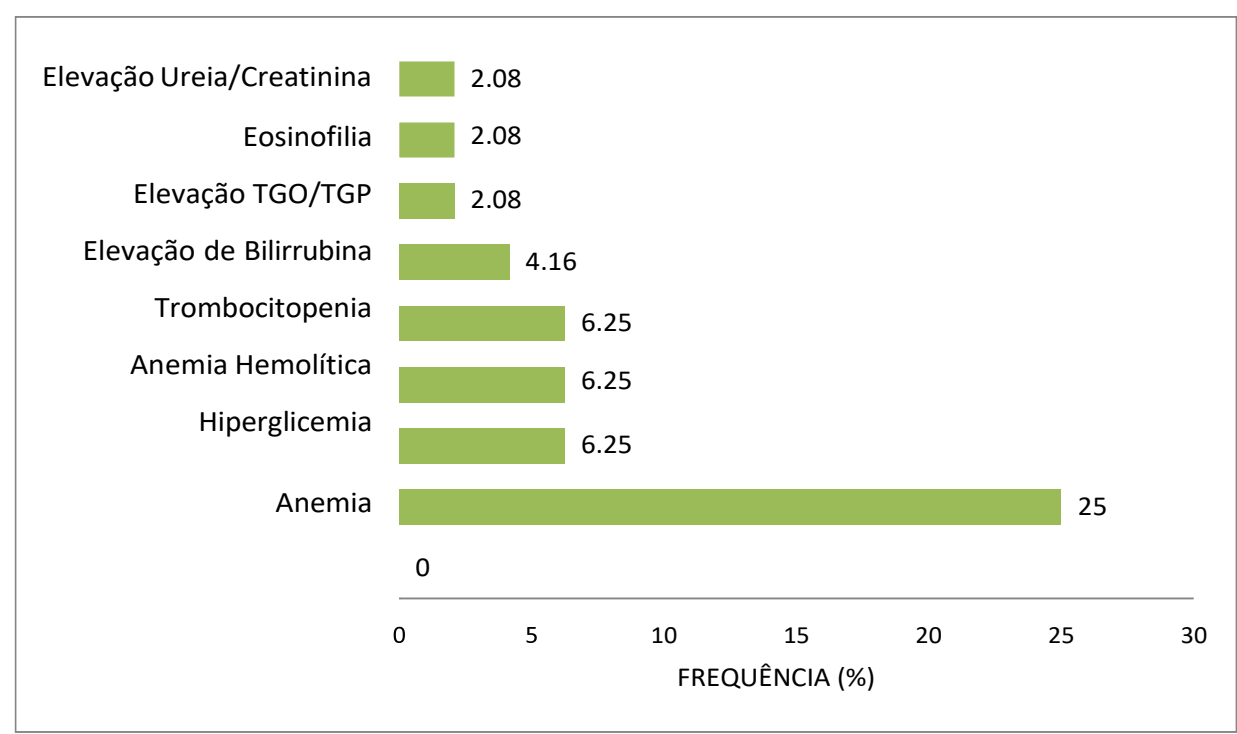

Fonte: Dados do estudo 2016.

No gráfico 1, podemos observar que dos eventos adversos laboratoriais 0 que teve maior frequência foi a anemia, presente em (25\%) pacientes, sendo que $(6,2 \%)$ pacientes apresentaram anemia hemolítica, alterações que, em geral, estão relacionadas à dapsona. Segundo os dados apresentados nos exames laboratoriais dos pacientes estudados, a variação de queda de Hemoglobina $(\mathrm{Hb})$ entre os pacientes com anemia foi de 0,7 a 2,7 $\mathrm{g} / \mathrm{dll}$, com média de $1,71 \mathrm{~g} / \mathrm{dl}$. A dapsona é apontada como principal causadora de efeitos indesejáveis, dentre as medicações utilizadas no tratamento para hanseníase, com destaque para anemia e efeitos gastrintestinais (LEMOS, 2013). 
Tabela 3: Efeitos adversos graves que levaram a mudança de esquema terapêutico.

\begin{tabular}{|c|c|c|c|c|}
\hline Efeitos adversos & $\mathbf{N}$ & $\begin{array}{c}\text { Droga } \\
\text { responsável }\end{array}$ & $\begin{array}{c}\text { Droga } \\
\text { substituta }\end{array}$ & $\begin{array}{c}\text { Esquema } \\
\text { terapêutico }\end{array}$ \\
\hline Metaemoglobinemia & 1 & Dapsona & Clofazim & PB \\
\hline Anemia hemolítica & 1 & Dapsona & Clofazimina & PB \\
\hline
\end{tabular}

Conforme a tabela $3,(4,16 \%)$ dos pacientes estudados necessitaram realizar a troca do esquema terapêutico utilizado, todos relacionados ao uso da dapsona. A mudança de esquema terapêutico ocorreu nos primeiros meses de tratamento. Para o MS, de fato, os efeitos adversos mais graves estão relacionados à dapsona, ocorrendo, geralmente, nas 6 primeiras semanas de tratamento (BRASIL, 2016).

Os efeitos adversos que causaram a suspensão do uso da dapsona foram a metaemoglobinemia, na forma leve, em 1 paciente, e anemia hemolítica em 1 paciente. Para o MS, na presença de anemia hemolítica, toma-se como conduta a suspensão do tratamento e avaliar se a ocorrência desse efeito está relacionada às doses autoadministradas de dapsona ou a dose supervisionada de rifampicina. Em caso de metaemoglobinemia leve, deve-se suspender 0 tratamento, ocorrendo regressão do quadro clínico, de forma gradual, após a suspensão (BRASIL, 2014).

Os efeitos adversos relacionados ao uso da rifampicina e da clofazimina não necessitaram de troca de esquema, por terem sido efeitos leves, sem comprometimento terapêutico (FRANCO, 2014).

Os esquemas terapêuticos substitutivos devem ser utilizados em casos em que há intolerância grave ou contraindicação a uma ou mais drogas da $P Q T$. No caso de intolerância a dapsona em pacientes $P B$, deve ser utilizado a clofazimina com doses diárias de 50mg autoadministradas e dose mensal supervisionada de $300 \mathrm{mg}$ (cápsulas de 100mg) (BRASIL, 2016). Nos dois pacientes que demandaram troca de esquema, a droga substituta foi a clofazimina (pacientes paucibacilares com intolerância a dapsona). 
Tabela 4 - Interferência das reações adversas na adesão do tratamento.

\begin{tabular}{lcr}
\hline Adesão ao tratamento & $\mathbf{n}(\%)$ & $\begin{array}{r}\text { Reações adversas como } \\
\text { causas de irregularidades }\end{array}$ \\
\hline Regular & $43(89,6)$ & --- \\
Irregular & $3(6,2)$ & 1 \\
Abandono & $2(4,2)$ & 2 \\
Total & $48(100 \%)$ & --- \\
\hline
\end{tabular}

Fonte: Dados do estudo 2016.

Na tabela 4, 43 (89,6\%) apresentaram boa adesão ao tratamento, e 3 $(6,2 \%)$ não compareciam de forma regular e $2(4,2 \%)$ pacientes abandonaram o tratamento. Dos 3 pacientes que compareceram irregularmente, 1 (33,3\%) estava associado ao surgimento de efeitos adversos durante o tratamento. Quanto aos pacientes que abandonaram o tratamento, os 2 casos estavam associados ao surgimento de efeitos adversos. É considerado caso de abandono de tratamento aquele em que o paciente não completou o tratamento dentro do prazo máximo estabelecido, embora tenha tido várias tentativas de retorno e continuação do tratamento (SOUSA et al., 2013).

A reação adversa que ocasionou irregularidade no tratamento de 1 dos pacientes foi a anemia hemolítica relacionada a dapsona. Os 2 pacientes que abandonaram o tratamento, justificaram como causa em um dos casos, a hiperpigmentação cutânea relacionada a clofazimina, e no outro caso, alterações gastrointestinais intensas, provavelmente associado a dose mensal supervisionada de rifampicina.

No estudo realizado por Franco (2014), sobre efeitos adversos na PQT para hanseníase, não foi encontrada nenhuma associação entre irregularidade do tratamento e surgimento de efeitos adversos, sugerindo o autor, que o fato de ter sido um estudo de cunho prospectivo, pode proceder com busca ativa de forma eficaz. Esse achado também justifica o fato de o presente estudo ter encontrado associação entre essas duas variáveis, pois se trata de um estudo 
retrospectivo, em que o paciente não pode ser acompanhado durante 0 tratamento.

Mesmo com os grandes avanços feitos nos últimos anos em relação ao tratamento com PQT para hanseníase, ainda faz-se necessário que se crie sistemas de vigilância de eventos adversos mais eficazes, esquemas terapêuticos mais eficientes, com menos efeitos adversos e menor duração de tratamento, além de busca ativa aos pacientes, para redução das taxas de abandono e irregularidade de tratamento.

\section{Conclusão}

A repercussão das reações adversas medicamentosas da PQT no andamento do tratamento de hanseníase envolve múltiplos fatores (epidemiológicos, demográficos, clínicos, e outros), que podem contribuir positiva ou negativamente no sucesso terapêutico.

Neste estudo, identifou-se nos registros dos prontuárioos que os efeitos adversos no tratamento da PQT foram pouco frequentes, sugerindo que possa haver subnotificação de tais efeitos nas anotações. É necessário que os profissionais de saúde estejam bem preparados para diagnosticar os efeitos adversos, efetuando o manejo clínico adequado com registro das informações. Outro ponto que pode ter influenciado no resultado foi a ausência de um instrumento adequado para se registrar as reações adversas.

Os efeitos adversos mais frequentes foram: efeitos cutâneos (ictiose/xerose, hiperpigmentação de pele) relacionados à clofazimina, efeitos adversos laboratoriais como a anemia, geralmente associados à dapsona, e efeitos gastrointestinais, associados às três drogas em uso na PQT.

Houve mudança de esquema terapêutico em dois pacientes, todos associados ao uso da dapsona, ocorrendo nos primeiros meses de tratamento. Este achado reforça a afirmação de que a dapsona é a droga mais associada a efeitos adversos graves, que necessitam de suspensão do uso.

Dois pacientes abandonaram 0 tratamento, todos relacionados ao surgimento de reações adversas, resultado este, apresentado em vários outros estudos. 
O fato de nem todos os prontuários estarem devidamente preenchidos, dificultou a realização da coleta de dados, comprometendo a utilização de certas variáveis (escolaridade, raça, comorbidades). Quanto às reações adversas, não foi encontrado exames laboratoriais anexados em alguns dos prontuários, ou ao menos, informações acerca dos resultados, sugerindo que algumas das reações tenham sido subnotificadas.

\section{Referencias}

BRASIL. Ministério da Saúde. Diretrizes para vigilância, atenção e eliminação da Hanseníase como problema de saúde pública: manual técnico-operacional [recurso eletrônico]. - Brasília: Ministério da Saúde, 2016.

BRASIL. Ministério da Saúde. Secretaria de Vigilância em Saúde. Guia de Vigilância em Saúde. Brasília: Ministério da Saúde, 2014.

BRASIL. Resolução № 466, de 12 de Dezembro de 2012. Conselho Nacional de Saúde, 2012. Disponivel em:

<http://www.conselho.saude.gov.br/resolucoes/2012/Reso466.pdf>. Acesso em: 14 Maio 2020.

BRASIL. Hanseníase: o que é, causas. sinais e sintomas, tratamento, diagnóstico e prevenção. Ministério da Saúde, 2019. Disponivel em: <https://saude.gov.br/saude-de-a-z/hanseniase>. Acesso em: 14 Maio 2020.

BRASIL. Ministério da Saúde. Sistema Nacional de Vigilância em Saúde. Relatório de situação: Paraíba. Brasília: Ministério da Saúde, 2011.

FRANCO, Lenise de Albuquerque. Reações adversas à poliquimioterapia em hanseníase. 201496 f. Dissertação (Dissertação em Ciências da Saúde) UFS. Aracajú. 
KAR, Sumit; PAL, Ranabir; BHARATI, Dharamvir Ranajan. Understanding noncompliance with WHO-multidrug therapy among leprosy patients in Assam, India. J Neurosci Rural Pract, v. 1, n. 1, p. 9-13, 2010.

LEMOS, Roberta Faria. Avaliação dos pacientes com hanseníase multibacilar submetidos ao esquema terapêutico substitutivo. $201351 \mathrm{f}$. Dissertação (Dissertação de Mestrado) - FIOCRUZ. Rio de Janeiro.

MAIA, Marina Valente; CUNHA, Maria da Graça Souza; CUNHA, Carolina Souza. Adverse effects of alternative therapy (minocycline, ofloxacin, and clofazimine) in multibacillary leprosy patients in a recognized health care unit in Manaus, Amazonas, Brazil. An Bras Dermatol;88(2):205-10. 2013.

MENDES, Andrezza de Oliveira. et al. Caráter clínico-epidemiológico e grau de incapacidade física nos portadores de hanseníase no município de Barbacena - MG e macrorregião no período de 2001 a 2010. Rev Med Minas Gerais; 24(4): 486-494. 2014.

MIRANZI, Sybelle de Souza Castro; PEREIRA, Lívia Helena de Morais; NUNES, Altacílio Aparecido. Perfil epidemiológico da hanseníase em um município brasileiro, no período de 2000 a 2006. Rev. Soc. Bras. Med. Trop; 43(1): 62-7. 2010.

ROQUE, Keroulay Estebanez; MELO, Enirtes Caetano Prates. Adaptação dos critérios de avaliação de eventis adversos a medicamentos para uso em um hospital público no Estado do Rio de Janeiro. Rev bras epidemiol, São Paulo, v. 13 , n. $4,2010$.

SOUSA, Adriana Alves. et al. A adesão ao tratamento da hanseníase por pacientes acompanhados em unidades básicas de saúde de Imperatriz-Ma. SANARE, Sobral, v. 12, n. 1, p. 06-12, jan/jun. 2013. 
SOUZA, Aieska; EL-ALZHARY, Rokea; FOSS, Norma Tiraboschi. Management of chronic diseases: an overwiew of the Brazilian governmental leprosy program. Int J Dermatol; 48: 109-116. 2009.

VIRMOND, Marcos da Cunha Lopes. Uma nova etapa para a eliminação da hanseníase como problema de saúde pública?. Rev. Salusv. (Online), v. 32, p. 3-8, 2013.

WHO. Estratégia mundial de eliminação da lepra 2016-2020: Acelerar a ação para um mundo sem lepra. 2016.

WHO. Global leprosy update, 2014: need for early case detection. Weekly Epidemiological Record; 90(36):461-76. 2015. 